\title{
A Study of Factors Affecting Renal Impairment in Patients of Trauma: A Prospective Non-Interventional Observational Study
}

\author{
Authors \\ Kamal Shukla $^{1}$, Geeta Ghag ${ }^{2}$, Siddhant Mathurvaishya ${ }^{3}$ \\ ${ }^{1}$ Assistant Professor, Department of General Surgery, RN Cooper Hospital and HHBT Medical College, \\ Mumbai \\ ${ }^{2}$ Associate Professor, Department of General Surgery, RN Cooper Hospital and HHBT Medical College, \\ Mumbai \\ ${ }^{3}$ Surgical Gastroenterology Resident, Asian Institute of Gastroenterology, Hyderabad \\ Corresponding Author \\ Dr Geeta Ghag \\ Associate Professor, Department of General Surgery, RN Cooper Hospital and HHBT Medical College, \\ Mumbai, India \\ Email: geetaghag1@gmail.com
}

\section{Introduction}

Acute renal failure (ARF) is a serious complication of trauma and has a high mortality. In large trauma population a low incidence of ARF is generally reported from $0.098 \%$ to $8.9 \%^{1}$. Retrospective studies focused only on those patients who developed ARF and required dialysis. Great importance for the development of ARF after trauma was attributed to pre-existing pathological conditions such as diabetes hypertension etc. But a prospective analysis of the risk factors especially in severely ill patients admitted in ICU is still lacking.

Acute kidney injury (AKI) is a rapidly progressive loss of renal function, generally characterized by oliguria (decreased urine production, quantified as less than $400 \mathrm{ml}$ per day in adults, less than 0.5 $\mathrm{mL} / \mathrm{kg} / \mathrm{h}$ in children or less than $1 \mathrm{~mL} / \mathrm{kg} / \mathrm{h}$ in infants); and fluid and electrolyte imbalance ${ }^{2}$.
In trauma patients, acute kidney failure usually occurs when the blood supply to the kidneys is suddenly interrupted or when the kidneys become overloaded with toxins. Causes of acute failure include accidents, injuries, or complications from surgeries in which the kidneys are deprived of normal blood flow for extended periods of time. Among the accidental causes of renal failure there is also crush syndrome when large amounts of toxins are suddenly released in the blood circulation after a long-compressed limb is suddenly relieved from the pressure obstructing the blood flow through its tissues, causing ischemia. The resulting overload can lead to the clogging and the destruction of the kidneys. It is a reperfusion injury that appears after the release of the crushing pressure.

An underlying cause must be identified and treated to arrest the progress, and dialysis may be necessary to bridge the time gap required for 
treating these fundamental causes. The alteration between oxygenation and energy demands as seen during trauma may induce damage to or death of tubular cells. The prospective characterization of risk factors of post traumatic ARF remains crucial and could help to reduce the ARF associated mortality.

\section{Aims and Objectives}

Acute renal failure is the most frequent cause of morbidity and mortality in trauma cases, it is often fatal and can be avoidable. So, inview of the above facts, this study was planned with the following Objectives

1) To study the renal impairments in patients of trauma.

2) To study the factors causing renal impairment

3) To study the outcomes of the renal impairment caused by the attributing factors

\section{Material and Methods}

The present study titled 'A Study of Factors Affecting Renal impairment in Patients of Trauma. A Prospective Non-Interventional Observational Study" is a prospective observational study carried out in Department of Surgery and trauma care center of a tertiary teaching hospital over a period of 1 year

Sample Size: 500 cases

\section{Inclusion Criteria}

1. All patients admitted in the trauma ward of tertiary care hospital including those who are on ventilatory support

2. Patients of any age

3. Patients of any sex

4. All patients giving informed consent

\section{Exclusion Criteria}

1. All patients those who are in a chronic renal failure state.

2. Patients of burn.

3. Pregnant females,
4. Critical and seriously injured patients who succumb within few hours of injury.

5. Language barrier existing even after the help of a translator,

6. Not giving consent to participate in the study.

7. Patients were admitted as per hospital protocols.

Patients underwent routine hematological investigations, chest $\mathrm{X}$ rays and USG.

All patients were examined and investigated according to pre-designed case record performa.

The study protocol was approved by the Institutional Ethics Committee. Informed consent was taken from all patients enrolled in the study.

\section{Observations and Results}

Total 500 patients were examined and studied during the study period of 1 year.

Out of 500 patients 411 were male and 89 females.

Table 1: Distribution of cases on the basis of sex and creatinine levels.

\begin{tabular}{|l|c|c|c|}
\hline \multirow{2}{*}{ Sex } & \multicolumn{2}{|c|}{ Rise in creatinine levels } & \multirow{2}{*}{ Total } \\
\cline { 2 - 3 } & Yes & No & \\
\hline Male & 83 & 328 & 411 \\
\hline Female & 13 & 76 & 89 \\
\hline Total & 96 & 404 & 500 \\
\hline
\end{tabular}

The above table (Table 1) shows that majority of the patients with deranged creatinine levels were male i.e. $86.46 \%$ of cases with deranged creatinine levels. The comparison between male and female population with deranged creatinine levels was statistically not significant.

Table 2: Distribution of cases on the basis of mode of injury.

\begin{tabular}{|l|c|c|}
\hline Mode of injury & Number of cases & Percentage \\
\hline Assault & 49 & 9.8 \\
\hline Fall & 116 & 23.2 \\
\hline Railway accidents & 115 & 23.0 \\
\hline Road traffic accidents & 220 & 44.0 \\
\hline Total & 500 & 100 \\
\hline
\end{tabular}

The above table (Table 2) shows that the majority of cases were due to road traffic accidents. 
Table 3: Distribution of the cases on the basis of mode of injury and creatinine levels

\begin{tabular}{|l|c|c|c|}
\hline \multirow{2}{*}{ Mode of injury } & \multicolumn{2}{|c|}{ Rise in creatinine levels } & \multirow{2}{*}{ Total } \\
\cline { 2 - 3 } & Yes & No & \\
\hline Assault & 7 & 42 & 49 \\
\hline Fall & 26 & 90 & 116 \\
\hline Railway accidents & 26 & 89 & 115 \\
\hline Road traffic accidents & 37 & 183 & 220 \\
\hline Total & 96 & 404 & 500 \\
\hline
\end{tabular}

Majority of the patients showing creatinine levels derangement were those injured due to road traffic accidents (Table 3 ).

Out of 500 cases, 196 cases were surgically operated and rest were conservatively managed. Out of 196 surgically operated cases, 43 showed altered creatinine levels. The comparison was not statistically significant.

Exposure to contrast medium was noted in 76 patients, out of which 22 had raised serum creatinine levels. $28.95 \%$ of total patients given contrast had deranged creatinine levels as compared to $17.7 \%$ who were not given. The comparison was significant with $\mathrm{p}$ value less than 0.05 .

Resuscitation was given to $88.8 \%$ (444/500) of the patients. $20.95 \%$ of the resuscitated patients had deranged creatinine levels, which implies that majority $(96.88 \%)$ of the patients with impaired creatinine levels required resuscitation.

Ventilatory support was required by 360 (72\%) patients. $24.44 \%(88 / 360)$ of the patients on ventilatory support had deranged creatinine levels. The number of patients having deranged creatinine levels and requiring ventilatory support was more than those having deranged creatinine levels but not requiring ventilatory support. The comparison was statistically significant with $p$ value less than 0.05 .

Hypovolemia was noted in $232(46.4 \%)$ cases. $30.17 \%$ (70/232) of hypovolemic patients had deranged creatinine levels, whereas among nonhypovolemic patients only $9.70 \%$ had deranged creatinine levels. $72.92 \%$ patients with raised creatinine levels were hypovolemic. The comparison was significant as $p$ value was less than 0.05 .
All 500 patients were followed up. Out of 500 patients 135 succumbed to the trauma, 182 patients were discharged and remaining 183 patients had increased duration (> 10 days) of stay in the hospital as duration of study was 10 days from date of admission to the hospital. $47.4 \%$ $(64 / 135)$ of dead patients had deranged renal profile. 30.21\% (29/96) patients with raised creatinine levels had extended duration of the stay.

Out of 500 patients, 210 had delay in presentation. 59 out of 96 patients with deranged renal function test had delay in presentation whereas remaining 37 were presented on time. The comparison between these two groups was statistically significant as observed $\mathrm{p}$ value was less than 0.05. Sepsis was noted in 135 cases out of 500 . Out of 135 cases of sepsis, 56(41.48\%) had impaired renal function. Among 365 non-sepsis cases only $39(10.68 \%)$ had deranged renal function test. The comparison of both the group was highly significant as $\mathrm{p}$ value was far less than 0.05 .

\section{Discussion}

Posttraumatic renal failure continues to be associated with a high morbidity and mortality.

However, the patient population is changing. Because of improved initial resuscitation techniques, the frequency of acute renal failure associated with lesser trauma has been reduced, whereas an increasing number of patients withmore severe trauma are surviving long enough for renal failure to become a major management problem. These patients frequently have many other complications including respiratory insufficiency, sepsis, liver failure, and coagulation abnormalities.

Lordon and Stone and Knepshield ${ }^{3}$ have recently reviewed the course ofpatients with renal failure following military trauma. Rapid resuscitation and evacuation to a definitive treatment center resulted in only a $0.2 \%$ incidence of renal failure in Vietnam. Mortality in those developing renal failure remained high (63\%; because of associated injuries and sepsis. Patients 
with lower genitourinary tract, thoraco-abdominal, brain, and abdominal injury had a higher mortality thanthe total patient population. Patients having chest injury without abdominal injury,or having head, face. and extremity injury had a lower mortality. The fatality rate could not be attributed to the management of renal failure, but was related to the extent of associated injuries. Sepsis was the most frequent cause of death.

In our series 500 patients were studied of which 96 patients showed creatinine derangements (19\%). Maximum patients were males [Table 1] between the age of $2 \mathrm{O}$ to 45 with RTA being the common mode of injury [Table 2].The comparison of gender data was not significant as the $\mathrm{p}$ value is more than 0.05 . In a study done by de Abreu $\mathrm{KL}^{4}$ on acute kidney injury after trauma using RIFLE classification showed the mean age involved was that of the age $30.1 \pm 19.2$ years, and $79.8 \%$ were males.

In our study, out of 96 patients having raised creatinine 30 patients were having pure head injuries which were severe in nature, majority having subdural hematoma with midline shift and were also associated with high mortality. When head injury was associated with other systems, mortality was also increased. Similar pattern of involvement was seen in a study done by James H. Sipkins ${ }^{5}$ who showed severe head trauma patients going in renal failure and ultimately requiring dialysis and patients with head injury with blunt abdomentrauma component having high mortality.

\section{Role of Hypovolemia}

Out of 500 patients of trauma 232 patients presented with hypovolemia on admission. The patients were treated with large volumes of Ringer's lactate and sodium bicarbonate until urine production was $60-100 \mathrm{ml} / \mathrm{hr}$. Hemoglobin and electrolytes were substituted according to the laboratory findings. There were (70/232) patients $(30.17 \%)$ showed increase in creatinine values. The comparison was significant as the $\mathrm{p}$ value observed was less than 0.05.It was observed that the renal failure did not develop during the initial 24-48 hrs. but during treatment in the intensive care unit, mostly in the event of multiple organ failure. Patients who presented with hypovolemia and eventually developed renal failure mainly presented with blunt abdominal trauma with hemoperitoneum with associated pelvic injury or patients with traumatic amputation and degloving injury having massive blood loss. A study done by U Finke $^{6}$ showed that immediate and rapid fluid correction can prevent renal failure in trauma patients which was found to be true in our study as well.

\section{Role of Surgery}

In our study 96 patients who developed creatinine derangements, 43 patients were operated and 53 patients were not operated. The comparison was not significant as the $\mathrm{p}$ value was more than 0.05 . Out of 43patients who got operated 38 patients expired and only 6 patients gone home.36out 43 patients who were operated they were having severe hypovolemia onadmission. This supports that operative stress alone is not responsible for the renal failure, but when combined with other risk factors predispose to the renal failure having high mortality rate.

Surgery imposes a significant stress on the body. Acute renal failure or worsening of an already established renal dysfunction is common during the peri-operative period. The renal damage is usually the result of multiple factors, but more frequently it is the result of an ischemic injury in a susceptible individual. Pre-existing renal disease is the single most important factor that predisposes individuals to worsening injury during the peri-operative period. Patients with medical conditions associated with decreased renal blood flow and low GFR, suchas diabetes mellitus, hypovolemia, dehydration and sepsis. Patients with cholestatic jaundice have a higher incidence of renal failure and post-op mortality. This may be due to a predisposition to hypotension due to low peripheral vascular resistance in cirrhotic patients, accompanying sepsis and associated disseminated 
intravascular coagulation. Predictive factors in these patients are the degree of bilirubin elevation and the degree of creatinine elevation. ${ }^{7}$ A review of the literature suggests that the most critical determinants of postoperative renal function are pre-operative renal function, maintenance of appropriate intravascular volume, and normal myocardial function. ${ }^{8}$

\section{Role of Contrast}

ln present study $28.95 \%$ of patients who received contrast agents for CT SCAN developed creatinine derangements. The comparison was significant as the $\mathrm{p}$ value was less than 0.05 . The incidencewas low because non-ionic contrast media was used and care was taken that precontrast creatinine value was normal $(<1.5 \mathrm{mg} / \mathrm{dl})$. If patients creatinine value was borderline raised, $\mathrm{N}$-ACETYLCYSTINE was given to prevent contrast induced nephropathy.

A study done by Robert T. Misson ${ }^{9}$ showed that the incidence of renalfailure/creatinine derangements post contrast infusion rises rapidly in those with azotemia from any cause, however, and diabetic persons with nephropathy areperhaps are at special risk. Vigorous volume expansion is possibly effective as apreventive measure and may attenuate adverse effects in those in whom post contrast dysfunction occurs. Gruberg et al ${ }^{10}$ published a retrospective observational study of 439 patients with renal insufficiency and showed: a $37.7 \%$ rate of $\mathrm{CIN}$ (contrast induced nephropathy).

\section{Role of Hemodialysis}

ln present study only (1/500) patient went hemodialysis who also expired after 7days. In a study done by Morris $\mathrm{JA} \mathrm{Jr}^{11}$ on Acute posttraumatic renal failure: amulticenter perspective at Vanderbilt University School of Medicine showed that Posttraumatic renal failure requiring hemodialysis is rare (incidence, 107 per100,000 trauma center admissions). but the mortality rate remains high $(57 \%)$.

\section{Role of Mechanical Ventilation}

Nearly all patients in our series showing creatinine derangements who ultimately went into renal failure were on ventilatory support. The comparison was significant as the $\mathrm{p}$ value was less than 0.05 . Vivino $G^{1}$ in his study showed that, the need for mechanical ventilation with a positive end-expiratory pressure $>6 \mathrm{~cm} \mathrm{H} 20$, is one of the condition most strongly associated with the renal failure. Similar results were present in the study of Kuiper $\mathrm{JW}^{12}$. The development of acute renal failure during mechanical ventilation likely represents a multifactorial process that may become more important in the presence of comorbidities.

\section{Role of Sepsis}

Sepsis is a major cause of mortality and morbidity in the trauma patient. Sepsis following traumatic injury is related to the type of injury, together with the extent of injury and the anatomical location. The diagnosis of sepsis in the trauma patient remains difficult. Interpretation of abnormal results is key to successful diagnosis, particularly in conjunction with clinical findings. In our study $58.33 \%$ (56/96) of patients with renal impairment were having sepsis. The comparison was significant as the $\mathrm{p}$ value was less than 0.05 $(<0.00001)$. Most of the patients with sepsis going in renal failure were of post laparotomy and degloving injury patients. The initial debridement of degloved wounds was carried out promptly. A good wound care was given to patients to help prevent infection in the first place. The wounds must be adequately explored washed and debrided as soon as possible after the patient's condition stabilizes. Robert W. Schrier ${ }^{13}$ in an article said Acute renal failure occurs in approximately $19 \%$ of patients with moderate sepsis, 23 percent with severe sepsis, and $51 \%$ with septic shock when blood cultures are positive. Hypovolemiain the presence of sepsis leads to circulatory collapse followed by renal failure. Continuous fluid therapy and accurate assessment of renal function in post trauma period is therefore very essential. 


\section{Role of NISS}

New injury severity score (NISS) is the modification of injury severity score (ISS). As multiple injuries within the same body region are only assigned a single score. A proposed modification of the ISS, the "New Injury Severity Score" (NISS), has been proposed. This is calculated as the sum of the squares of the top three scores regardless of body region. The NISS has been found to be statistically outperform the traditional ISS score. In our study $88.54 \%$ of the patients (85/96) with impaired renal function were having NISS of $>17$.The higher the NISS score more were the chances of mortality observed. In a study conducted by Vivino $G^{1}$ the risk of post traumatic acute renal failure increases if ISS score is $>17$. Study conducted by Tran DD et $\mathrm{al}^{14}$ also showed higher ISS to be a causative factor of acute renal failure in severe civilian trauma patients.

\section{Delay in Presentation}

Delay in presentation is associated with other factors like hypovolemia due to prolonged blood loss, high injury severity score, hemoperitoneum was a causative factor for renal impairment in our series of patients. Railway accident patients having blunt chest trauma, blunt abdominal trauma who were referred from the periphery hospitals to our tertiary care hospital were the potential candidates. The comparison between the delay of presentation and rise of creatinine was significant since the $\mathrm{p}$ value observed was less than 0.05 .

\section{Conclusion}

Five hundred patients of trauma were analyzed and ninety-six were having raised creatinine. Creatinine level derangements were seen mostly in patients of polytrauma who presented with severe hypovolemia and who presented late for the treatment. Sepsis and ventilatory support were also shown to be the causative factor for creatinine level derangement as their comparison with creatinine levels were significant and $\mathrm{p}$ value was shown to be less than 0.05. Early identification of these factors and their timely correction can thus prevent renal failure in trauma patient which has high mortality.

\section{References}

1. Vivino G. M. Antonellie. Risk factors for acute renal failure in traumapatients. Intensive Care Med. 1998 Aug;24(8):80814.

2. Schrier RW, Wang W, Poole B, Mitra A (2004). "Acute renal failure:definitions, diagnosis, pathogenesis, and therapy". J. Clin. Invest. 114(1):5-14

3. Smith LH. Post-traumatic renal insufficiency in military casualties. II. Management, use of an artificial kidney. prognosis. Am J Med.1955; 18:187-198

4. De Abreu KL. Acute kidney injury after trauma: Prevalence. Clinical characteristics and RIFLE classification. lndian J Crit Care Med. 2010 Jul;14(3):121-8

5. Sipkins JH, Kjellstrand CM: Severe Head Trauma and Acute Renal Failure. Nephron 1981; 28:36-41.

6. U Finke Aggressive volume therapy for prevention of acute renalfailure In multiple trauma patients Volume: 69, Suppl 26, Pages;119 - 123.

7. Novis BK, Roizen MF, Aronson S: Association of perioperativerisk factors with postoperative acute renal failure. AnesthAnalg 1994; 78:143-149.

8. Kellerman PS. Perioperative care of the renal patient. Arch InternalMed 1994; 154: 1674-88

9. Misson RT, Cutler RE: Radiocontrastinduced renal failure [MedicalProgress]. West J Med 1985 May; 142:657-664

10. Gruberg L, Mintz GS, Mehran R, et al: The prognostic implications offurther renal function deterioration within $48 \mathrm{~h}$ of interventional coronaryprocedures in patients with pre-existent chronic renal 
insufficiency. J AmColl Cardiol 2000;36 (5):1542-1548

11. Morris JA Department of Surgery. Vanderbilt University School ofMedicine Nashville, Tennessee. 1991 Dec;31 (12):1584-90.

12. Kuiper JW, Mechanical ventilation and acute renal failure.Criti CareMed. 2005 Jun;33(6):1408-15

13. Wilson R., Schrier N. Acute Renal Failure and Sepsis, Engl J Med 2004;351:159-169

14. Tran DD. Acute renal failure in patients with severe civilian trauma.Nephrol Dial Transplant. 1994;9(Suppl 4):121—5. 